\title{
Avaliação da atividade antioxidante e teor de compostos fenólicos em extrato metanólico obtido de folhas da Commiphora Myrrha
}

\section{Evaluation of antioxidant activity and phenolic compounds content in methanol extract obtained from leaves Commiphora Myrrha}

\author{
Celia Eliana de Lara da Silva ${ }^{1}$; Rosilene Valota ${ }^{2}$; Karimi Sater Gebara ${ }^{3}$; Rogério Cesar \\ de Lara da Silva'; Euclésio Simionatto ${ }^{5}$
}

\section{Resumo}

O presente trabalho mostra o estudo da atividade antioxidante e quantificação do teor de compostos fenólicos determinados para o extrato metanólico obtido da Commiphora myrrha. O alto teor de compostos fenólicos foram avaliados frente ao seu potencial em seqüestrar radical livre por meio do modelo 2,2-difenil-1-picrizil hidrazil (DPPH) e comparados ao um padrão de rutina. O resultados mostram que a capacidade inibitória do extrato $\left(\mathrm{IC}_{50}\right)$ foi de $0,21 \mathrm{mg} \cdot \mathrm{L}^{-1}$. O extrato exerceu uma atividade antioxidante de $91,3 \%$ quando comparados a capacidade sequestradora do padrão de rutina. $\mathrm{O}$ teor de compostos fenólicos do extrato foi avaliado pelo uso do reagente de Folin-Ciocalteu onde o $\mathrm{IC}_{50}$ determinado foi de $3,02 \mathrm{mg} . \mathrm{L}^{-1}$. A concentração de fenóis totais determinada foi de 1,176 \pm $0,263 \mathrm{mg}$ equivalente de ácido gálico por $\mathrm{g}$ de extrato $(\mathrm{n}=5)$. Os resultados mostram que os extratos da C. myrrha possuem alto potencial antioxidante e estudos adicionais são necessários para isolamento, caracterização e uso de suas propriedades em aplicações farmacêuticas, nutricionais e cosmetológica.

Palavras-Chave: Atividade antioxidante. Compostos fenólicos. DPPH. $\mathrm{IC}_{50}$

\begin{abstract}
This work presents shows the study of antioxidant activity and quantification of phenolic content determined for the methanol extract obtained from Commiphora myrrha. The high content of phenolic compounds were evaluated against the potential to sequester free radical through the model 2,2-diphenyl1-picrizil hydrazyl (DPPH) and compared with a standard rutin. The results show that the inhibitory capacity of the extract $\left(\mathrm{IC}_{50}\right)$ was $0.21 \mathrm{mg} . \mathrm{L}^{-1}$. The extract pursued an antioxidant activity of $91.3 \%$ compared to the scavenging ability of rutin standard. The content of phenolic extract was assessed by using the Folin-Ciocalteu determined where the $\mathrm{IC}_{50}$ was $3,02 \mathrm{mg} . \mathrm{L}^{-1}$. The concentration of total phenols was determined $1.176 \pm 0.263 \mathrm{mg}$ gallic acid equivalent. $\mathrm{g}^{-1}$ of extract $(\mathrm{n}=5)$. The results show that extracts of C. myrrha have high antioxidant potential and additional studies are needed for isolation, characterization and use of their property in pharmaceutical, nutritinal and cosmetology.
\end{abstract}

Keywords: Antioxidant activity. Phenolic compounds. DPPH. IC $\mathrm{I}_{50}$

${ }^{1}$ Especialista do Centro Universitário da Grande Dourados - UNIGRAN. Departamento: Especialização em Saúde Pública. Dourados - MS - CEP - 79824-900.

${ }^{2}$ Especialista do Centro Universitário da Grande Dourados - UNIGRAN. Departamento: Especialização em Saúde Pública. Dourados - MS - CEP - 79824-900.

${ }^{3}$ Docente Mestre do Centro Universitário da Grande Dourados - UNIGRAN. Departamento: Especialização em Saúde Pública. Dourados - MS - CEP - 79824-900.

${ }^{4}$ Docente da Universidade Estadual de Mato Grosso do Sul - UEMS. Química Licenciatura. Departamento de Química. Naviraí - MS - CEP: 79950-000.

${ }^{5}$ Docente da Universidade Estadual de Mato Grosso do Sul - UEMS. Química Licenciatura. Departamento de Química. Naviraí - MS - CEP: 79950-000 


\section{Introdução}

Moléculas instáveis que possuem radicais livres quando presentes em organismos vivos podem levar a um processo de estresse oxidativo, causando danos as células e patologias relacionadas como artrite, câncer, diabete, doenças cardíacas e neurodegenerativas, dentres outras (AYRES; CHAVES, 2009). Espécies radicalares como o radical hidroxila $(\cdot \mathrm{OH})$, ânion radical superóxido $\left(\mathrm{O}_{2} \bullet^{-}\right)$e o hidroperoxila $(\mathrm{ROO} \bullet)$ podem oxidar lipídios e proteínas assim como causar danos ao DNA e oxidar cadeias de moléculas de ácidos graxos poliinsaturados dos fosfolipídios e de colesterol (VELLOSA et al., 2007).

A minimização destes efeitos em nosso organismo pode ser feita através da ingestão de antioxidantes naturais, principalmente através de nossa dieta alimentar e outras fontes como suplementos alimentares. Alguns dos principais agentes antioxidantes são os tocoferóis (vitamina E), o ácido ascórbico (vitamina C), os polifenóis, selênio e carotenóides (HASLAM, 1996; VALKO et al., 2004). Muitos dos antioxidantes são encontrados em plantas na forma de compostos fenólicos tais como fenóis simples, ácidos fenólicos derivados de ácidos benzóico e cinâmico, cumarinas, flavonóides, etc., (NACZK; SHAHIDI, 2004). Os antioxidantes são capazes de estabilizar ou desativar os radicais livres antes que ataquem os alvos biológicos nas células. São comumente encontrados em frutas, legumes, verduras e também em plantas de uso medicinal ou de uso popular sendo obtidos extratos através do uso de solventes orgânicos (SOUZA et al., 2007).

A Commiphora myrrha (Nees) Engl., da família Burseraceae, do gênero Commiphora, possui mais de 200 espécies (VOLLESEN, 1989). O óleo da C. myrrha é o único que apresenta odor aromático doce e picante, diferente das demais espécies que são amargos, sendo a classe dos terpenos os compostos mais característicos e responsáveis pelo cheiro (FRATERNALE et al., 2011). A resina e o óleo extraído da myrrha são muito valorizados na
Ásia, sendo fonte de renda para muitas pessoas, além de seu uso medicinal para inúmeras doenças, desde alergias, infecções genitais e problemas respiratórios (ORWA et al., 2009). Na China é extensamente utilizada como planta medicinal pela população, para tratar várias doenças, como inflamações de boca, dores estomacais, amenorréia, feridas, tumores, doenças inflamatórias, cicatrizante e dor (SHEN et al., 2008). Um dos constituintes mais abundantes da resina da $C$. myrrha são os furanos e sesquiterpenos (MORTEZA-SEMNANI; SAEEDI, 2003). É popularmente conhecida como mirra, muito utilizada desde a antiguidade como aromatizante, sendo originária da África, principalmente Somália, Etiópia, e Arábia Saudita (BASER et al., 2003; HANUS et al., 2005). Esta espécie possui facilidade de crescimento em solo raso e pode ser utilizada para controle de erosão. O teor de óleo essencial pode variar de 3 a $8 \%$ (TUCKER, 1986), sendo este muito estudado em países asiáticos, como a China. Marongiu et al. (2005) comparou a extração do óleo por hidrodestilação contra a de fluído supercrítico e obteve rendimentos semelhantes entre os métodos além de sua composição semelhante em análise por espectrometria de massas. Ensaios feitos em ratos revelaram a capacidade analgésica e antiinflamatória, fundamentando o uso tradicional para este fim (SU et al., 2011). Shena et al., (2009), estudando resinas de exsudatos de árvores da C. myrrha, isolaram e identificaram por técnicas espectroscópicas, RMN e ESI-MS novos triterpenoides e sesquiterpenoides. A atividade citotóxica dos compostos isolados foi avaliada contra células tumorais de próstata PC3 e DU145. Helal et al. (2005), realizando experimentos em ratos machos com extrato aquoso da C. myrrha conseguiram reduzir a glicemia, estimulando a produção das células $\mathrm{B}$ e aumento na produção de insulina, no entanto relatam que necessitam de estudo mais detalhado em níveis diferentes. Pesquisas de $\mathrm{Su}$ et al. (2011) demonstram a atividade citotóxica de compostos diterpênicos, isolados da fração ativa do extrato da C. myrrha, em células humanas cancerígenas do aparelho 
ginecológico. Conseguiram isolar nove compostos químicos que seriam responsáveis pela inibição do crescimento dessas células. Zhu et al. (2001, 2003), em pesquisas do exsudato da goma da C. myrrha, isolaram três novos compostos sesquiterpenóides. Esses compostos apresentaram pouca ou nenhuma atividade citotóxica contra células tumorais de mama. Como a C. myrrha já tem suas propriedades medicinais reconhecidas tanto popularmente como cientificamente, por não haver estudos com os extratos de suas folhas e pela importância e carência de se descobrir antioxidantes naturais, justifica-se o presesnte estudo.

A atividade antioxidante a partir de extratos obtidos de folhas da myrrha não tem sido explorada nas pesquisas apresentadas.

\section{Materiais e Métodos}

\section{Reagentes e equipamentos}

Para avaliação do potencial da atividade antioxidante foram utilizados o radical livre 2,2-difenil-1-picril-hidrazila (DPPH) e o padrão de rutina como controle positivo para comparação, todos da Aldrich Co. O reagente Folin-Ciocalteu foi obtido, e o ácido gálico para uso de quantificação foram obtidos da Vetec e Merck, respectivamente. O metanol (PA) foi obtido da Cynth, para preparação do extrato e de soluções.

As análises foram realizadas no laboratório de pesquisa do curso de Química Licenciatura da Universidade Estadual de Mato Grosso do Sul (UEMS) na cidade de Naviraí - MS. Espectrofotômetro UV-visível da Varian - Cary 50 , com cela de quartzo de $1 \mathrm{~cm}$ de largura, foi utilizado nas medidas de absorbância das amostras analisadas.

\section{Material vegetal}

Folhas do material vegetal $C$. myrrha foram coletadas no Horto Florestal da cidade de Naviraí
- MS, no mês de maio de 2011, e transportado ao laboratório de química orgânica da UEMS. As folhas foram secas a temperatura ambiente para perda de umidade, e posteriormente acondicionada em frasco de vidro com metanol e lacradas para obtenção do extrato. Após um período de 15 dias, o metanol foi evaporado em evaporador rotativo e o extrato concentrado seco à temperatura ambiente.

\section{Avaliação da atividade antioxidante}

Aavaliação quantitativa da atividade antioxidante do extrato metanólico obtido foi realizada perante o consumo do radical livre DPPH preparado em concentração de $0,004 \%(\mathrm{~m} / \mathrm{V})$, através de medidas de absorbâncias de soluções em diferentes concentrações do extrato, segundo metodologia Rosler et al. (2007), Souza et al. (2007) e Silva et al. (2009). Solução estoque do extrato seco foi preparada em metanol a uma concentração de 400 $\mathrm{mg} \mathrm{L}^{-1}$ para sucessivas diluições. A partir da solução estoque alíquotas de $0,1 \mathrm{~mL}, 0,2 \mathrm{~mL}, 0,4 \mathrm{~mL}, 0,6 \mathrm{~mL}$, $0,8 \mathrm{~mL}$ e $1,0 \mathrm{~mL}$ foram acondicionadas em balões volumétricos de $10 \mathrm{~mL}$ contendo $2 \mathrm{~mL}$ da solução do radical livre DPPH $(0,004 \%)$ e completados com metanol resultando em concentrações do extrado de 4,0 mg. $\mathrm{L}^{-1}$ à $40,0 \mathrm{mg} \cdot \mathrm{L}^{-1}$. Após um período de reação de 30 minutos foram realizadas leituras em espectrofotômetro UV-vis em $517 \mathrm{~nm}$ para determinação da absorbância do DPPH. Uma solução estoque de 2 g.L $\mathrm{L}^{-1}$ do padrão de rutina, utilizado como controle positivo foi preparada para sucessivas diluções em concentrações conhecidas (40,0 mg. $\mathrm{L}^{-1}$ a 400,0 mg.L $\mathrm{L}^{-1}$ ) para comparação do percentual de inibição frente ao extrato analisado. Uma medida do branco foi obtido através da mistura do metanol mais o reagente DPPH. O percentual do seqüestro de radical livre foi calculado pela seguinte equação:

$$
\% A A=\left(\frac{A_{0}-A}{A_{0}}\right) \times 100
$$

onde \%AA é o percentual da atividade antioxidante, $A_{0}$ é a absorbância do DPPH utilizado como controle e $A$ é absorbância da amostra mais DPPH. 
O \%AA corresponde a quantidade de DPPH consumida pelo antioxidante, sendo esta a quantidade necessária para decrescer a concentração inicial de DPPH em 50\% é chamada de concentração inibitória $\left(\mathrm{IC}_{50}\right)$.

\section{Quantificação do teor de fenóis}

O teor de fenóis totais foi determinado pelo método de Folin-Ciocalteu (BRASIL, 2010; SINGLETON; ROSSI, 1965). Uma solução estoque do ácido gálico de concentração 1 g.L. $\mathrm{L}^{-1}$ em água foi preparada para sucessivas diluições. Construção de curva analítica para o ácido gálico foi realizada em concentrações de $0,5 \mu \mathrm{g} \cdot \mathrm{mL}^{-1}$ a $6,0 \mu \mathrm{g} \cdot \mathrm{mL}^{-1}$ por sucessivas diluições. A estas soluções preparadas foram adicionados $0,4 \mathrm{~mL}$ do reagente FolinCiocalteu, seguidos de $1,2 \mathrm{~mL}$ de $\mathrm{Na}_{2} \mathrm{CO}_{3} 20 \%$. Depois de decorrido a reação por um período de $1 \mathrm{~h}$ e 30 min. a absorbância das amostras foi realizada em espectrofotômetro UV-vis em $750 \mathrm{~nm}$. Solução estoque em metanol do extrato seco de 1 g.L.- foi preparada para novas diluições. A partir desta solução, $200 \mu \mathrm{L}$ foram adicionados em balões de $10 \mathrm{~mL}$ seguidos da adição de $1,2 \mathrm{~mL}$ do $\mathrm{Na}_{2} \mathrm{CO}_{3}$ e $0,4 \mathrm{~mL}$ do reagente de Folin-Ciocalteu. Um total de cinco amostras foi analisado para determinação do teor de fenóis sendo este expresso em mg por equivalente de ácido gálico por grama de extrato (mg EAG.g ${ }^{-1}$ ).

\section{Resultados e Discussão}

A atividade antioxidante medida através do radical estável DPPH, mostra a capacidade de substâncias pertencentes ao extrato da $C$. myrrha obtido em seqüestrar radicais livres do meio existente. É uma metodologia simples, rápida, e eficiente na avaliação de extratos obtidos a partir de solventes com diferentes polaridades. Os testes realizados para analisar a atividade antioxidante do extrato metanólico obtido das folhas da C. myrrha utilizando o DPPH e comparado ao padrão de rutina são descritos na Tabela 1 a seguir.

Tabela 1 - Atividade antioxidante obtida para o extrato da C. myrrha e padrão de rutina.

\section{Extrato C. myrrha}

\begin{tabular}{c|c|c}
\hline C.E. $\left(\mathbf{m g}^{-1} \mathbf{L}^{-1}\right)$ & $\mathrm{Abs}^{1}$ & \% AA \\
\hline 4,0 & 0,201 & 82,9 \\
\hline 8,0 & 0,188 & 84,0 \\
\hline 16,0 & 0,149 & 87,3 \\
\hline 24,0 & 0,120 & 89,8 \\
\hline 40,0 & 0,039 & 96,7 \\
\hline
\end{tabular}

Padrão de rutina

\begin{tabular}{c|c|c}
\hline C. $\left.\mathbf{R}_{\text {( }} \mathbf{m g . L ^ { - 1 }}\right)$ & $\mathbf{A b s}^{2}$ & \% AA \\
\hline 40,0 & 0,071 & 94,7 \\
\hline 80,0 & 0,040 & 97,0 \\
\hline 200,0 & 0,040 & 97,0 \\
\hline 400,0 & 0,038 & 97,2 \\
\hline
\end{tabular}

C.E. - Concentração do extrato da C. myrrha; ${ }^{1}$ Abs do branco do DPPH 1,175 para análise do extrato metanóico; \% AA- percentual de atividade antioxidante; C.R. - concentração do padrão de rutina; ${ }^{2}$ Abs do branco do DPPH 1,351 para análise com padrão de rutina como controle positivo.

Fonte: Autor 
As substâncias existentes no extrato possuem a capacidade em reagir com o radical livre instável do DPPH e convertê-lo no 2,2-difenil1-picril hidrazina. A capacidade ou a atividade de atuar como um antioxidante é visível quando da descoloração das soluções preparada. $\mathrm{O} \mathrm{IC}_{50}$, correspondente a concentração do extrato da planta necessária para inibir $50 \%$ da atividade do radical livre, foi calculado por meio da análise da regressão linear entre a C.E. vs \%A.A. gerando uma equação de reta de $\mathrm{y}=383,6 \pm 13,1 \mathrm{x}+81,1 \pm 0,3$ onde $y$ representa a \%A.A. e $x$ a concentração do extrato, com r de 0,9965 na qual o $\mathrm{IC}_{50}$ determinado foi de $0,21 \mathrm{mg} \cdot \mathrm{L}^{-1}$. A atividade antioxidante do padrão de rutina utilizado como controle positivo, apresentou uma média de 96,5\%, considerando este valor como $100 \%$, o extrato da C. Myrrha apresentou uma média de $88,1 \%$, exercendo 91,3 $\%$ do potencial antioxidante da rutina.

A atividade antioxidante para extratos de folhas da $C$. myrrha não são reportadas em literatura. Entretanto, extratos de folhas de outras espécies do gênero Commiphora (ex. C. schimperi, C. neglecta, C. tenuipetiolata, C. edulis, C. berryi e C. caudata) exibem atividade antioxidante frente a ensaios com DPPH onde o $\mathrm{IC}_{50}$ determinados permaneceram entre 7,31 e 26,92 mg.. $\mathrm{L}^{-1}$ (PARASKEVA et al., 2008, KUMARI et al., 2011). Estes valores de $\mathrm{IC}_{50}$ estão acima do determinado para o extrato da $C$. myrrha $\left(0,21 \mathrm{mg} . \mathrm{L}^{-1}\right)$. A atividade antioxidante para óleo de algumas espécies do gênero Commiphora são mostradas. Segundo Racine e Auffray (2005), o óleo essencial exibi atividade antioxidante devido a anéis de furano (particulamente de furanosesquiterpenos) os quais reagem com radicias livres. Estudos de Fraternale et al., (2001), com o óleo da myrrha demostram que os furanosesquiterpenos exibem atividade sequestradora de radicais livres frente ao DPPH onde o $\mathrm{EC}_{50}$ determinado foi de 1,08 a 4,29 $\mathrm{mg} . \mathrm{L}^{-1}$ para tres compostos isolados e identificados. Segundo Fraternale et al., (2011), realizando a hidrodestilação de folhas da C. erythraea para extração do óleo, a atividade sequestradora de radical pelo uso do DPPH determinou-se o $\mathrm{EC}_{50}$ com $14,28 \mathrm{mg} \cdot \mathrm{mL}^{-1}$.

O teor de fenóis para o extrato metanólico foi avaliado pelo reagente de Folin-Ciocalteu, o qual tem sido extensamente usado na determinação de diversos extratos. O reagente é constituído dos ácidos fosfomolibídico e fosfotunguístico. Estes sofrem redução na presença de compostos fenólicos de extratos formando colorações azuladas da solução devido à redução do estado de oxidação dos metais molibdênio e tungstênio para +5 . Através da reação entre o ácido gálico e o reagente de Folin-Ciocalteu foi determinada a análise de regressão linear, com equação da reta y $=0,1453 \pm 0,0111 \mathrm{x}+0,06 \pm 0,0345$ com coeficiente de correlação $r$ igual á 0,9769 . A Figura 1 mostra a reta determinada em faixa de concentração de 0 a $6,0 \mathrm{mg} . \mathrm{L}^{-1}$, onde o $\mathrm{IC}_{50}$ determinado para o teor de fenóis totais foi de 3,02 mg. $\mathrm{L}^{-1}$. Um total de cinco amostras do extrato foram analisadas com o reagente de Folin-Ciocalteu e obteve-se uma concentração de fenóis totais de 1,176 $\pm 0,263 \mathrm{mg}$ EAG . $\mathrm{g}^{-1}$ com um desvio padrão relativo de $6,4 \%$ entre as medidas. A análise do teor de fenois para óleo e extratos de folhas não são reportados para as espécies do gênero Commiphora em literatura. Entretanto observa-se que o teor de fenóis para o extrato metanólico obtido foi baixo quando comparado a outras plantas medicinais. Hossain et al., (2011), estudando extratos orgânicos de folhas da planta medicinal Tetrastigma sp., obteve para o extrato metanólico 386,22 mg EAG . g-1 . Souza et. al., (2007), analizando extrato etanólico de cinco plantas medicinais encontradas no cerrado (Terminalia brasiliensis Camb., Terminalia fagifolia Mart. \& Zucc., Copernicia cerifera (Miller) H.E. Moore, Cenostigma macrophyllum Tul. var. acuminata Teles Freire e Qualea grandiflora Mart) permaneceram entre 250 e 763 mg EAG $\cdot \mathrm{g}^{-1}$. 
Figura 1 - Curva analítica do ácido gálico para quantificação de fenóis totais

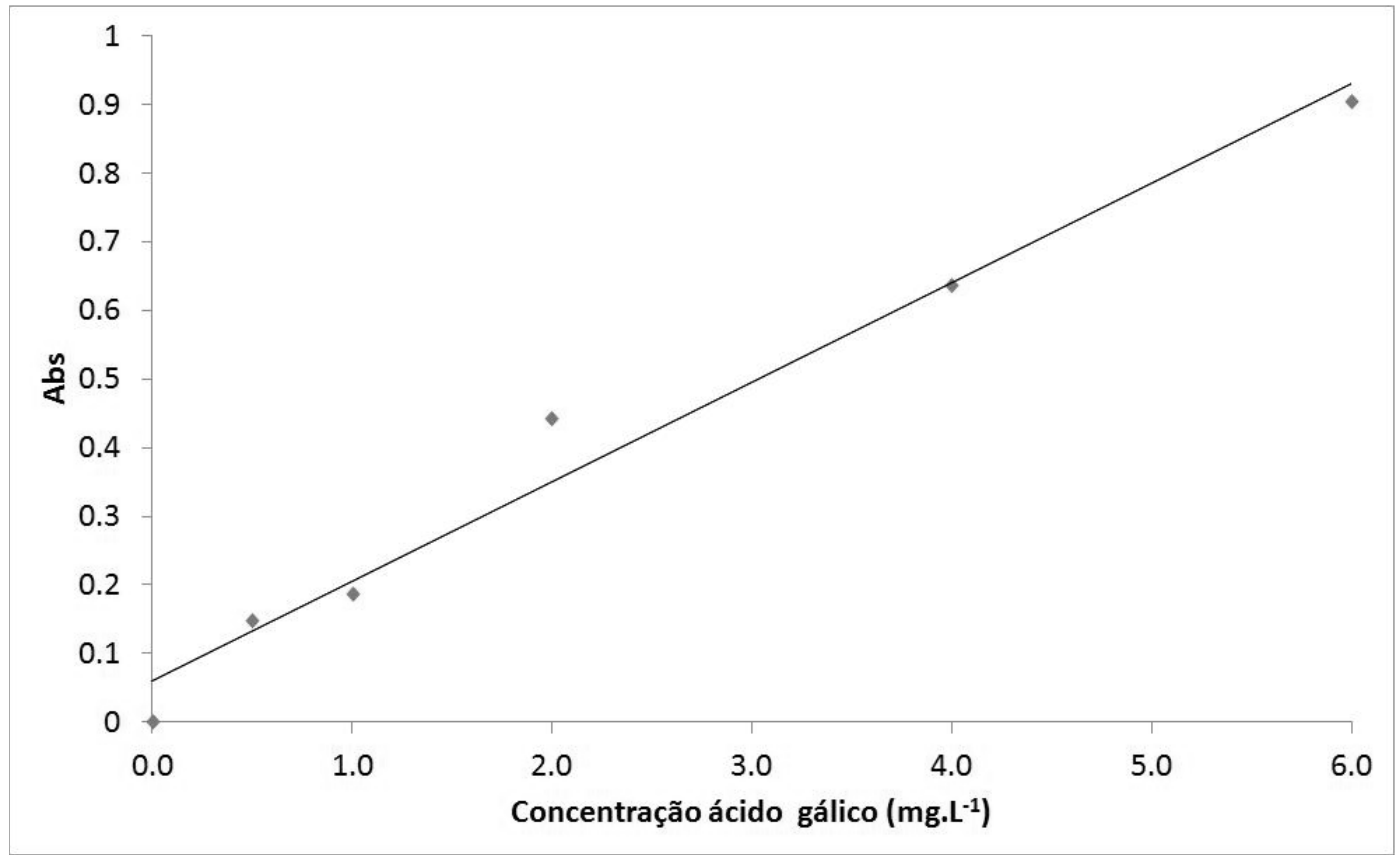

Fonte: Autor

\section{Considerações Finais}

Por meio dos resultados obtidos, conclui-se que extrato metanólico de folhas da C. myrrha possue boa capacidade de sequestrar radicais livres atuando como um atioxidante natural. Entretanto, mais estudos relacionado a estapas de isolamento, e caracterização dos compostos fenólicos responsáveis pela atividade antioxidante são necessários. Aplicações economica em áreas farmacêutica, cosmetologia e nutricionais podem ser exploradas.

\section{Referências}

AYRES, M. C. C.; CHAVES, M. H. Constituintes químicos e atividade antioxidante de extratos das folhas de Terminalia Fagifolia Mart. Et Zucc. Química Nova, São Paulo, v. 32, n. 6, p. 1509-1512, 2009.

BASER, K. H. C.; DEMIRCI, B.; DEKEBO, A.; DAGNE, E. Essential oils of some Boswellia ssp. myrrh and opopanax. Flavour and Fragrance Journal, Chichester, v. 18, p. 153-156, 2003.
BRASIL. Farmacopeia brasileira: agência nacional de vigilância sanitária. Brasília: ANVISA, 2010. v. 2.

FRATERNALE, D.; SOSA, S.; RICCI, D.; GENOVESE, S.; MESSINA, F.; TOMASINI, S.; MONTANARI, F.; MARCOTULLIO, M. C. Anti-inflammatory, antioxidant and antifungalfuranosesquiterpenoids isolated from Commiphora erythraea (Ehrenb.) Engl. Resin. Fitoterapia, Milano, v. 82, p. 654-661, 2011.

HANUS, L. O.; REZANKA, T.; DEMBITSKY, V. M.; MOUSSAIEFF, A. Myrrh: Commiphora chemistry. Biomedical Papers, Czechoslovakia, v. 149, n. 1, p. 3-28, 2005.

HASLAM, E. Natural polyphenols (vegetable tannins) as drugs: possible modes os action. Journal of Natural Products, Cincinnati, v. 59, n. 2, p. 205-215, 1996.

HELAL, E. G. E.; MAHMOUD, A.; EL-BADAWY, E. E.; KAHWASH, A. A. Effect of Commiphora myrrha extract on some physiological parameters and histological changes in diabetic albino rats. The Egyptian Journal of Hospital Medicine, Cairo, v. 20, p. 148-162, 2005.

HOSSAIN, M A.; MUHAMMAD, D. S.; GNANARAJ, C.; IQBAL, M. In vitro total phenolics, flavonoids contents and antioxidant activity of essential oil, various organic extracts from the leaves of tropical medicinal plant Tetrastigma from Sabah. Asian Pacific Journal of Tropical Medicine, Singapore, v. 4, n. 9, p. 717-721, 2011. 
KUMARI, R.; MEYYAPPAN, A.; NANDI, D.; AGRAWALLA, B. K.; CHOWDHURY, A. A.; SELVAMANI. P.; LATHA, S.; GIRI, V. S.; MUKHERJEE. J.; BANDYOPADHYAY, S.; JAISANKAR, P. Antioxidant and antibacterial activities of bark extracts from Commyphora berryi and Commiphora caudata. Natural Product Reseach, London, v. 25, p. 1454-1462, 2011.

MARONGIU, B.; PIRAS, A.; PORCEDDA, S.; SCORCIAPINO, A. Chemical Composition of the Essential Oil and Supercritical CO2 Extract of Commiphora myrrha (Nees) Engl. and of Acorus calamus L. Journal of Agricultural and Food Chemistry, Easton, v. 53, p. 7939-7943, 2005.

MORTEZA-SEMNANI, K.; SAEEDI, M. Constituents of the Essential Oil of Commiphora myrrha (Nees) Engl. var. molmol. Journal of Essential Oil Research, Carol Stream, v. 15, p. 50-51, 2003.

NACZK, M.; SHAHIDI, F. Extraction and analysis of phenolics in food. Journal of Chromatography A, Amsterdam, v. 1054, n. 1/2, p. 95-111, 2004.

ORWA, C.; MUTUA, A.; KINDT, R.; JAMNADASS, R.; SIMONS, A. Agroforestree database: a tree reference and selection guide version 4.0. 2009. Disponivel em: $<$ http://www.worldagroforestry.org/af/treedb/>. Acesso em: 12 jun. 2012.

PARASKEVA, M. P.; VUUREN, S. F.; ZYL, R. L.; DAVIDS, H.; VILJOEN, A. M. The in vitro biological activity of selected South African Commiphora species. Journal of Ethnopharmacology, Lausanne, v. 119, p. 673-679, 2008.

PIETTA, P. G. Flavonoids as antioxidants. Journal of Natural Products, Cincinnati, v. 63, p. 1035-1042, 2000.

RACINE, P; AUFFRAY, B. Quenching of singlet molecular oxygen by Commyphora myrrha extracts and menthofuran. Fitoterapia, Milano, v. 76, p. 316-323, 2005.

ROSLER, R.; MALTA, L. G.; CARRASCO, L. C.; HOLANDA, R. B.; SOUZA, C. A. S.; PASTORE, G. M. Atividade antioxidante de frutas do cerrado. Ciência e Tecnologia de Alimentos, Campinas, v. 27, n. 1, p. 53-60, 2007.

SHEN, T.; YUAN, W. Z.; WAN, X. L.; WANG, X. N.; WANG, M.; JI, M.; LOU, H. X. Cycloartane-type triterpenoids from the resinous exudates of Commiphora opobalsamum. Journal of Natural Products, Cincinnati, v. 71, n. 1, p. 81-86, 2008 .

SHENA, T.; WANA, W.; WANGA, X.; YUANB, H.; JIA, M.; LOU, H. A Triterpenoid and Sesquiterpenoids from the Resinous Exudates of Commiphora myrrha. Helvetica Chimica Acta, Basel, v. 92, n. 4, p. 645-652, 2009.
SILVA, A. C.; OLIVEIRA, M. C.; DEL RÉ, P. V.; JORGE, N. Utilização de extrato de cogumelo como antioxidante natural em óleo vegetal. Ciência e Agrotecnologia, Lavras, v. 33, n. 4, p. 1103-1108, 2009.

SINGLETON, V. L.; ROSSI, S. A. Colorimetric of total phenolics with phosphomolibic-phosphotungstic acid reagents. American Journal of Enology \& Viticulture, California, US, v. 16, p. 144-158, 1965.

SOARES, S. E. Ácidos fenólicos como antioxidantes. Revista de Nutrição, Campinas, v. 15, n. 1, p. 71-81, 2002.

SOUSA, C. M. M.; SILVA, H. R.; JUNIOR, G. M. V.; AYRES, M. C. C.; COSTA, C. L. S.; ARAÚJO, P. B. M.; BRANDÃO, M. S.; CHAVES, M. H. Fenóis totais e atividade antioxidante de cinco plantas medicinais. Química Nova, São Paulo, v. 30, n. 2, p. 353-355, 2007.

SU, S.; WANGB, T.; DUANA, J.; ZHOUA, W.; HUAA, Y.; TANGA, Y.; YUA, L.; QIANA, D. Anti-inflammatory and analgesic activity of different extractsof Commiphora myrrha. Journal of Ethnopharmacology, Lausanne, v. 134, p. 251-258, 2011.

TUCKER, A. O. Frankincense and myrrh. Economic Botany, Bronx, v. 40, p. 425-433, 1986.

VALKO, M.; IZAKOVIC, M.; MAZUR, M.; RHODES, C. J.; TELSER, J. Role of oxygen radicals and DNA damage and cancer incidence. Molecular and Cellular Biochemistry, Holanda, v. 266, n. 1/2, p. 37-56, 2004.

VELLOSA, J. C. R.; BARBOSA, V. F.; KHALIL, N. M.; SANTOS, V. A. F. F. M.; FURLAN, M.; LOURENÇO, I.; OLIVEIRA, O. M. M. F. Profile of Maytenus aquifolium action over free radicals ande reactive oxygen species. Brazilian Journal of Pharmaceutical Sciences, São Paulo, v. 43, n. 3, 2007.

VOLLESEN, K. Burseraceae. In: HEDBERG, I., EDWARDS, S. (Ed.). Flora of Ethiopia. Uppsala: Addis Ababa University Press, 1989. v. 3, p. 442-478.

ZHU, N.; KIKUZAKI, H.; SHENG, S.; SHENG, S.; RAFI, M. M.; WANG, M.; NAKATANI, N.; DIPAOLA, R. S.; ROSEN, R. T.; HO, C-T. Furanosesquiterpenoids of Commiphora myrrha. Journal of Natural Products, Cincinnati, v. 64, p. 1460-1462, 2001.

ZHU, N.; SHENG, S.; SANG, S.; ROSEN, R. T.; HO, C. T. Isolation and characterization of several aromatic sesquiterpenes from Commiphora myrrha. Flavour and Fragrance Journal, Chichester, v. 18, p. 282-285, 2003.

Recebido em 10 Fevereiro 2012- Received on February 10, 2012.

Aceito em 13 Dezembro, 2012 - Accepted on December 13, 2012. 
